\title{
Human Impacts on Coastal Communities : A Case Study of Benthic Faunal Changes after Dike Construction in Saemangeum, South Korea
}

\author{
Shin'ichi Sato*1, a, Hiroyoshi Yamashita*2, Kyungwon Kim*3 \\ and Masatoshi Matsuo*4
}

\begin{abstract}
Major reclamation projects have resulted in large impacts on the natural environment of coastal areas worldwide. We investigated faunal changes in benthos following the construction of reclamation dikes in the area of Saemangeum, on the western coast of South Korea, and compared them with those in Isahaya Bay, Japan. The world's largest reclamation dike was constructed in Saemangeum. Following its completion in April 2006, the tidal flats in this area gradually dried out, and we confirmed that numerous dead shells of mollusks and other benthic animals were exposed on the dried tidal flat in June 2006. The inner part of Isahaya Bay was isolated from the rest of Ariake Bay by a reclamation dike in April 1997. The intertidal zone of this bay also dried out completely within several months, and numerous dead shells were exposed on the dried mud flats. Changes in benthic fauna were also confirmed in the outer part of Isahaya Bay. Red tides and hypoxic water have frequently been observed, and the numbers of many bivalve species have decreased rapidly since 1997. Similar changes have already been confirmed in the outer parts of the Saemangeum dike. These facts suggest that the Saemangeum dike will negatively affect the Yellow Sea in the near future.
\end{abstract}

Keywords : benthic fauna, reclamation dike, Isahaya Bay, Saemangeum area, South Korea

\section{Introduction}

Recent industrialization has greatly affected coastal communities worldwide. Along the coast of Asia, such anthropogenic effects are especially remarkable in tidal flats and estuarine environments, in which many benthic animals are threatened with extinction (e.g. Wada et al., 1996 ; Hong, 2000 ; Sato and Koh, 2004). Detailed descriptions of such artificial changes in benthic communities are useful for understanding the mechanisms of faunal response to large- scale environmental changes that have occurred in the geological past.

Extensive tidal flats occur around the western coast of South Korea facing the Yellow Sea. There are many ongoing reclamation projects in these tidal flats (Hong, 2000 ; Sato and Koh, 2004). One of the largest projects is the Saemangeum Reclamation Project (Fig. 1-A). The length of the dike is $33 \mathrm{~km}$, which is the largest in the world, and the reclamation area is 401 $\mathrm{km}^{2}$, which is more than 10 times larger than that of the Isahaya Reclamation Project in Ja-

Received November 19, 2006. Accepted March 8, 2007.

*1 The Tohoku University Museum. 6-3 Aoba, Aramaki, Aoba-ku, Sendai, 980-8578, Japan.

*2 Association of Conservation Malacology. 3-1-26-103 Kugenuma-Matsugaoka, Fujisawa, 251-0038, Japan.

*3 Korean Federation for Environmental Movement. 251 Nuha-dong, Jongno-gu, Seoul 110-806, South Korea.

*4 Graduate School of Science and Technology, Nagasaki University. 1-14 Bunkyo-machi, Nagasaki, 852-8521, Japan.

*a Corresponding author : kurosato@mail.tains.tohoku.ac.jp 
pan (Fig. 1-B, C). The construction of the dike began in November 1991. The northern part of this dike was built in June 2003, and the southern part of the dike was finally completed in April 2006. Although the water gates have not yet been closed completely, the tidal range inside the dike decreased dramatically after the completion of the dike. As a result, the intertidal zone inside the dike gradually dried out within several months, and numerous dead shells and crabs were exposed on the dried tidal flats (Hong et al., 2007).

The inner part of Isahaya Bay, Japan, was similarly isolated from the rest of Ariake Bay by the construction of a dike in April 1997, and most of the tidal mud flat area, about $29 \mathrm{~km}^{2}$, has been lost (Fig. 1-C). Following this isolation, all benthic animals inhabiting the tidal flats were killed as a result of desiccation and starvation in the inner parts of the dike (Sato, 1997, 2000a, b, 2001). In the subtidal zone of the inner bay (currently an adjustment pond), the salinity of the bottom water decreased suddenly to $<5 \mathrm{psu}$ within 4 months of isolation, and the bivalve and gastropod fauna was altered dramatically (Sato et al., 2001 ; Sato and Azuma, 2002). Moreover, the environmental conditions changed suddenly in the outer part of Isahaya Bay, and the bivalve fauna was replaced on a massive scale (Sato and Kanazawa, 2004 ; Kanazawa et al., 2005).

The scale of these reclamation areas is large enough to be comparable to an environmental change that occurred in the geological past. For example, the closing of a lagoon or embayment by growing sand bars is a similar phenomenon to that of the dike construction described here. Beginning in May 2000, we conducted periodic quadrat sampling along two transects on tidal flats in the Saemangeum area and investigated the changes in the benthic community over the following 6 years. Here, we report the benthic faunal changes in response to the large environmental disturbance in Saemangeum and compare them with those in Isahaya Bay.
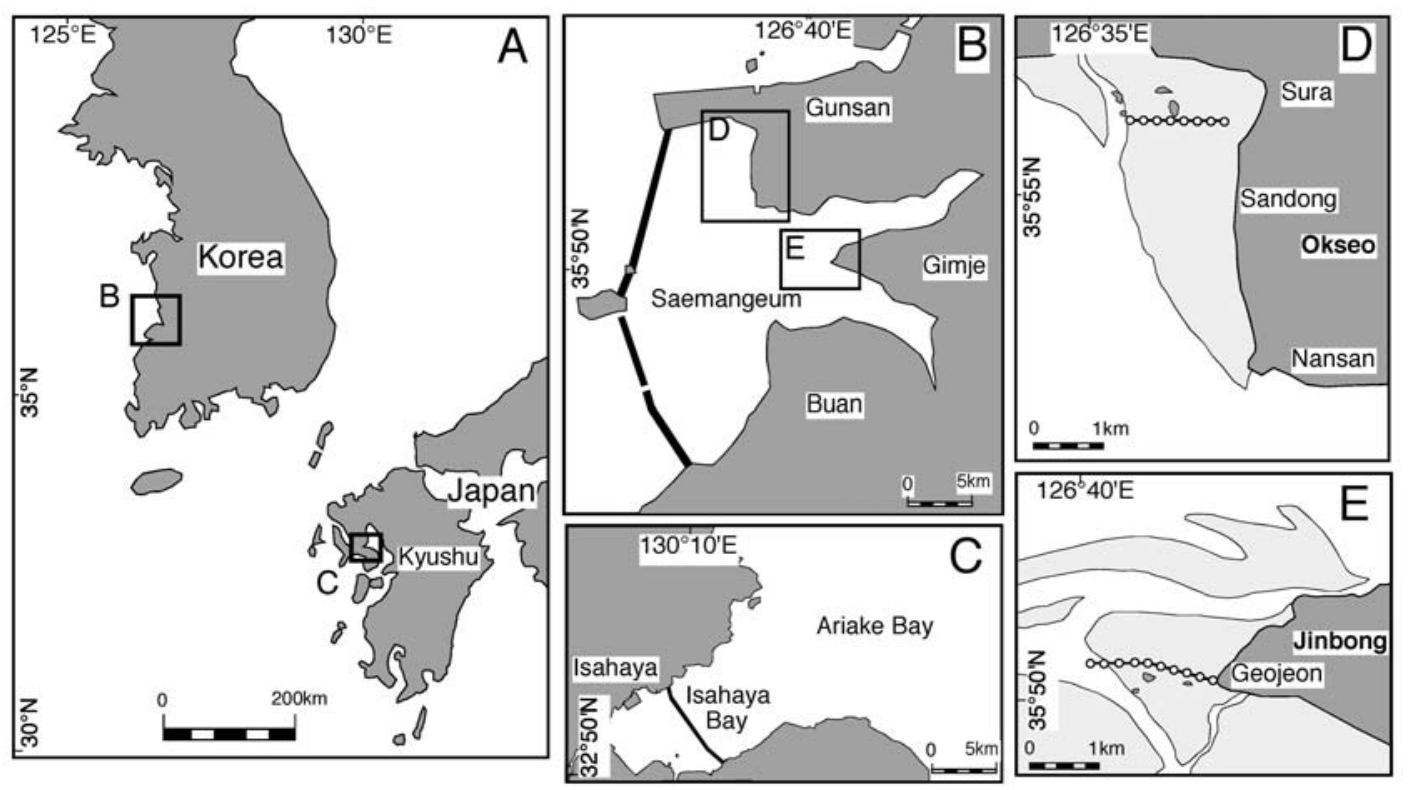

Fig. 1 Locations of extensive reclamation projects in Korea and Japan

A : Index map, B, C : Location of the reclamation area in (B) Saemangeum, Korea, and (C) Isahaya Bay, Japan. Black bars represent dikes completed in each area. Maps of $\mathrm{B}$ and $\mathrm{C}$ are drawn at the same scale. D, E : Location of (D) eight sampling stations on the tidal flat around Okbong-ri, Okseo-myeon, Gunsan City and (E) ten sampling stations on the tidal flat around Simpo-ri, Jimbong-myeon, Gimje City. Dark gray areas indicate land; light gray areas indicate tidal flats. 


\section{Materials and methods}

Sediment samples were collected from eight fixed stations on the tidal flats around Okbong-ri, Okseo-myeon, Gunsan City (Fig. 1-D), and ten stations on the tidal flat around Simpo-ri, Jimbong-myeon, Gimje City (Fig. 1-E), from May 2000 to September 2006 (see Table 1). A transect was established in each tidal flat, and the sampling stations were fixed at $200 \mathrm{~m}$ intervals from land (Fig. 1-D, E). Each sampling station was located using a global positioning system (GPS) receiver (Empex : Pocketnavi Mini), and one or two quadrat samples $(25 \times 25 \mathrm{~cm})$ were dug to a depth of $20 \mathrm{~cm}$ using a shovel and passed through a net with about $2 \mathrm{~mm}$ mesh size.

Many polychaetous annelids passed through the $2 \mathrm{~mm}$ mesh, and it was impossible to accurately count their numbers. Therefore, we did not count numbers of individuals of polychaetous annelids. Moreover, when many individuals of the brachiopod Lingula occur within an area, it is difficult to collect all individuals because they usually live more than $20 \mathrm{~cm}$ in depth from the surface of the tidal flat. We collected as many individuals as possible, but the numbers of Lingula may have been underestimated. Also, by June and September 2006, two and five stations, respectively, near land around Okbongri had already become dry, so living animals were observed visually on the dry tidal flat without sieving (Table 1).

The salinity of the surface water was measured using a hand-held salinity refractometer (Iuchi Co., Ltd. ; S-10) at channels near sampling station 8 (St. 8), 1,400 m from land. The remnants of sediment samples were preserved in ethanol, and living benthic animals were removed from the debris and identified in the laboratory. The samples collected from the Saemangeum area will be stored at the National Biological Resources Center in South Korea.

\section{Results}

In both tidal flats around Okbong-ri and Simpori, the salinity of the surface water near St. 8 was about 30 psu in winter and spring and 15$20 \mathrm{psu}$ in summer and fall (Fig. 2). In the Saemangeum area, the salinity of the surface water did not decrease dramatically between June and September 2006, because the water gates remained open after dike construction was completed. Nevertheless, numerous dead shells were exposed on the dried tidal flat because the tidal range inside the dike decreased dramatically after completion of the dike. In particular, in

Table 1 Locality, sampling date, and number of samples collected at each station

(1) Okbong-ri, Okseo-myeon, Gunsan City

\begin{tabular}{|c|c|c|c|c|c|c|c|c|c|c|c|c|c|}
\hline \multirow{2}{*}{$\begin{array}{c}\text { Station } \\
\text { No. }\end{array}$} & \multirow{2}{*}{$\begin{array}{l}\text { Distance } \\
\text { from land } \\
\text { (m) }\end{array}$} & \multirow{2}{*}{$\begin{array}{l}\text { Latitude } \\
\left(35^{\circ} \mathrm{N}\right)\end{array}$} & \multirow{2}{*}{$\begin{array}{c}\text { Longitude } \\
\left(126^{\circ} \mathrm{E}\right)\end{array}$} & \multicolumn{10}{|c|}{ Number of quadrat samples collected from each station } \\
\hline & & & & $\begin{array}{l}\text { May } \\
2000\end{array}$ & $\begin{array}{l}\text { Aug. } \\
2002\end{array}$ & $\begin{array}{l}\text { May } \\
2003\end{array}$ & $\begin{array}{l}\text { Sep. } \\
2003\end{array}$ & $\begin{array}{l}\text { Oct. } \\
2004 \\
\end{array}$ & $\begin{array}{l}\text { May } \\
2005\end{array}$ & $\begin{array}{l}\text { Dec. } \\
2005\end{array}$ & $\begin{array}{l}\text { Mar. } \\
2006 \\
\end{array}$ & $\begin{array}{l}\text { June } \\
2006 \\
\end{array}$ & $\begin{array}{l}\text { Sep. } \\
2006\end{array}$ \\
\hline 3 & 400 & $55^{\prime} 05.9^{\prime \prime}$ & $36^{\prime} 19.2^{\prime \prime}$ & 2 & 2 & 1 & 1 & 1 & 1 & 0 & 1 & $1^{*}$ & $1^{*}$ \\
\hline 4 & 600 & $55^{\prime} 05.9^{\prime \prime}$ & $36^{\prime} 11.2^{\prime \prime}$ & 1 & 0 & 1 & 1 & 1 & 1 & 0 & 1 & $1^{*}$ & $1^{*}$ \\
\hline 5 & 800 & $55^{\prime} 05.9^{\prime \prime}$ & $36^{\prime} 03.2^{\prime \prime}$ & 1 & 1 & 1 & 1 & 1 & 1 & 0 & 1 & 1 & $1^{*}$ \\
\hline 6 & 1000 & $55^{\prime} 05.9^{\prime \prime}$ & $35^{\prime} 55.2^{\prime \prime}$ & 1 & 0 & 1 & 1 & 1 & 1 & 0 & 1 & 1 & $f^{*}$ \\
\hline 7 & 1200 & $55^{\prime} 05.9^{\prime \prime}$ & $35^{\prime} 47.2^{\prime \prime}$ & 1 & 1 & 1 & 1 & 1 & 1 & 0 & 1 & $t$ & $1^{*}$ \\
\hline 8 & 1400 & $55^{\prime} 05.9^{\prime \prime}$ & $35^{\prime} 39.2^{\prime \prime}$ & 1 & 1 & 1 & 1 & 1 & 1 & 0 & 1 & 1 & 1 \\
\hline 9 & 1600 & $55^{\prime} 05.9^{\prime \prime}$ & $35^{\prime} 31.2^{\prime \prime}$ & 1 & 1 & 1 & 1 & 1 & 1 & 0 & 1 & 0 & 0 \\
\hline 10 & 1800 & $55^{\prime} 05.9^{\prime \prime}$ & $35^{\prime} 23.2^{\prime \prime}$ & 1 & 1 & 1 & 1 & 1 & 1 & 0 & 1 & 0 & 0 \\
\hline \multicolumn{14}{|c|}{ (2) Simpo-ri, Jinbong-myeon, Gimje City } \\
\hline \multirow{2}{*}{$\begin{array}{c}\text { Station } \\
\text { No. }\end{array}$} & \multirow{2}{*}{$\begin{array}{l}\text { Distance } \\
\text { from land } \\
(\mathrm{m})\end{array}$} & \multirow{2}{*}{$\begin{array}{l}\text { Latitude } \\
\left(35^{\circ} \mathrm{N}\right)\end{array}$} & \multirow{2}{*}{$\begin{array}{c}\text { Longitude } \\
\left(126^{\circ} \mathrm{E}\right)\end{array}$} & \multicolumn{10}{|c|}{ Number of quadrat samples collected from each station } \\
\hline & & & & $\begin{array}{l}\text { May } \\
2000 \\
\end{array}$ & $\begin{array}{l}\text { Aug. } \\
2002 \\
\end{array}$ & $\begin{array}{l}\text { May } \\
2003 \\
\end{array}$ & $\begin{array}{l}\text { Sep. } \\
2003\end{array}$ & $\begin{array}{l}\text { Oct. } \\
2004\end{array}$ & $\begin{array}{l}\text { May } \\
2005 \\
\end{array}$ & $\begin{array}{l}\text { Dec. } \\
2005\end{array}$ & $\begin{array}{l}\text { Mar. } \\
2006\end{array}$ & $\begin{array}{c}\text { June } \\
2006 \\
\end{array}$ & $\begin{array}{l}\text { Sep. } \\
2006\end{array}$ \\
\hline 2 & 200 & $50^{\prime} 32.4^{\prime \prime}$ & $40^{\prime} 55.0^{\prime \prime}$ & 2 & 1 & 1 & 1 & 1 & 1 & 1 & 1 & 1 & 1 \\
\hline 3 & 400 & $50^{\prime} 41.0^{\prime \prime}$ & $40^{\prime} 45.1^{\prime \prime}$ & 1 & 1 & 1 & 1 & 1 & 1 & 1 & 1 & 1 & 1 \\
\hline 4 & 600 & $50^{\prime} 41.9^{\prime \prime}$ & $40^{\prime} 35.1^{\prime \prime}$ & 1 & 1 & 1 & 1 & 1 & 1 & 1 & 1 & 1 & 1 \\
\hline 5 & 800 & $50^{\prime} 43.8^{\prime \prime}$ & $40^{\prime} 35.0^{\prime \prime}$ & 1 & 1 & 1 & 1 & 1 & 1 & 1 & 1 & 1 & 1 \\
\hline 6 & 1000 & $50^{\prime} 47.3^{\prime \prime}$ & $40^{\prime} 16.7^{\prime \prime}$ & 1 & 1 & 1 & 1 & 1 & 1 & 1 & 1 & 1 & 1 \\
\hline 7 & 1200 & $50^{\prime} 48.3^{\prime \prime}$ & $40^{\prime} 09.4^{\prime \prime}$ & 1 & 1 & 1 & 1 & 1 & 1 & 1 & 1 & 1 & 1 \\
\hline 8 & 1400 & $50^{\prime} 48.3^{\prime \prime}$ & $40^{\prime} 01.4^{\prime \prime}$ & 1 & 0 & 1 & 1 & 1 & 1 & 1 & 1 & 1 & 1 \\
\hline 9 & 1600 & $50^{\prime} 48.3^{\prime \prime}$ & $39^{\prime} 53.4^{\prime \prime}$ & 1 & 1 & 1 & 1 & 1 & 1 & 1 & 1 & 1 & 1 \\
\hline 10 & 1800 & $50^{\prime} 48.3^{\prime \prime}$ & $39^{\prime} 45.4^{\prime \prime}$ & 1 & 1 & 1 & 1 & 1 & 1 & 1 & 1 & 1 & 1 \\
\hline 11 & 2000 & $50^{\prime} 48.3^{\prime \prime}$ & $39^{\prime} 37.4^{\prime \prime}$ & 0 & 0 & 1 & 1 & 1 & 1 & 1 & 1 & 1 & 0 \\
\hline
\end{tabular}

* Living animals were collected from the dried tidal flat without sieving. 
the tidal flat around Okbong-ri, the stations within $600 \mathrm{~m}$ of land were dried out in June 2006, and those within $1,200 \mathrm{~m}$ of land were dried completely in September 2006 (Table 1).

\section{A. Okbong-ri, Gunsan City}
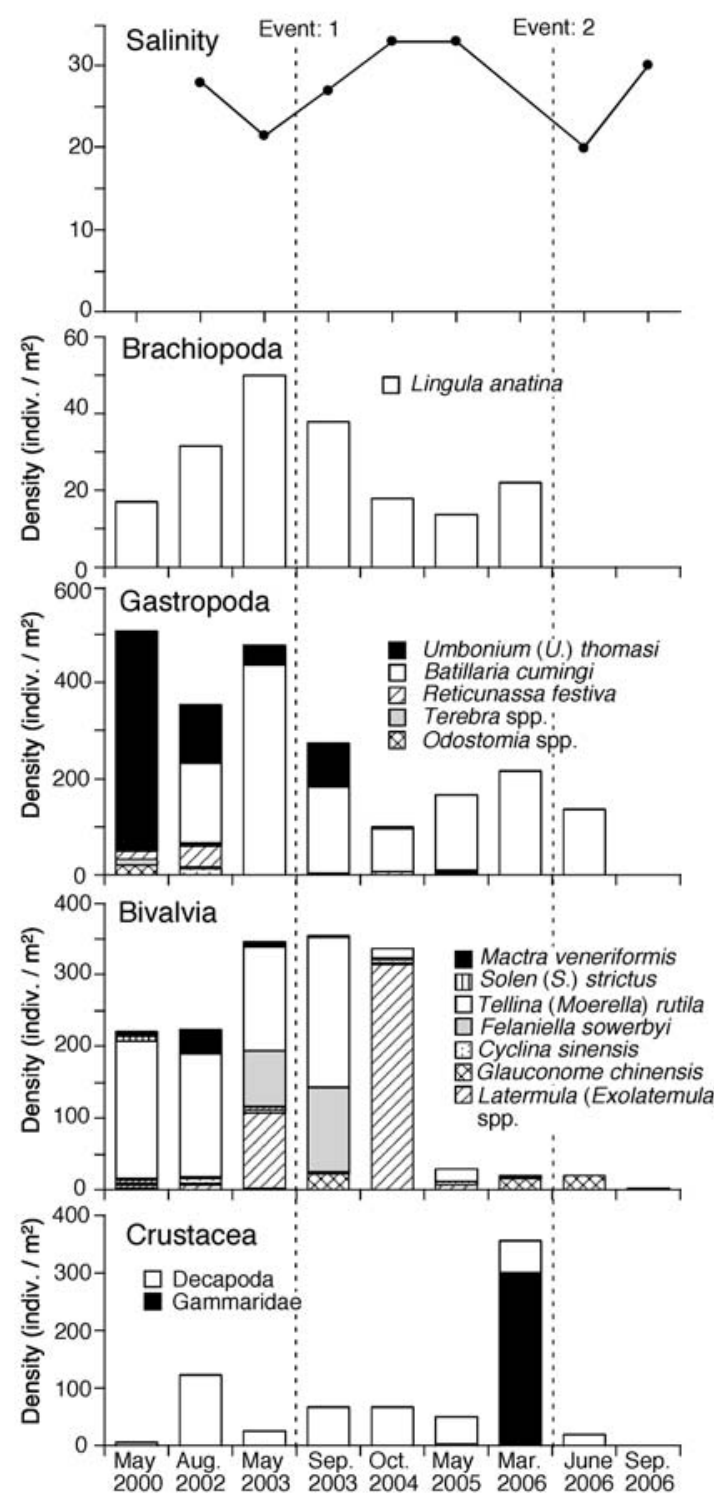

In May 2000, the brachiopod Lingula anatina Lamarck (1801), six species of gastropods such as Umbonium (U.) thomasi (Crosse, 1863), Reticunassa festiva (Powys, 1833) and Bullacta exarata

\section{B. Simpo-ri, Gimje City}
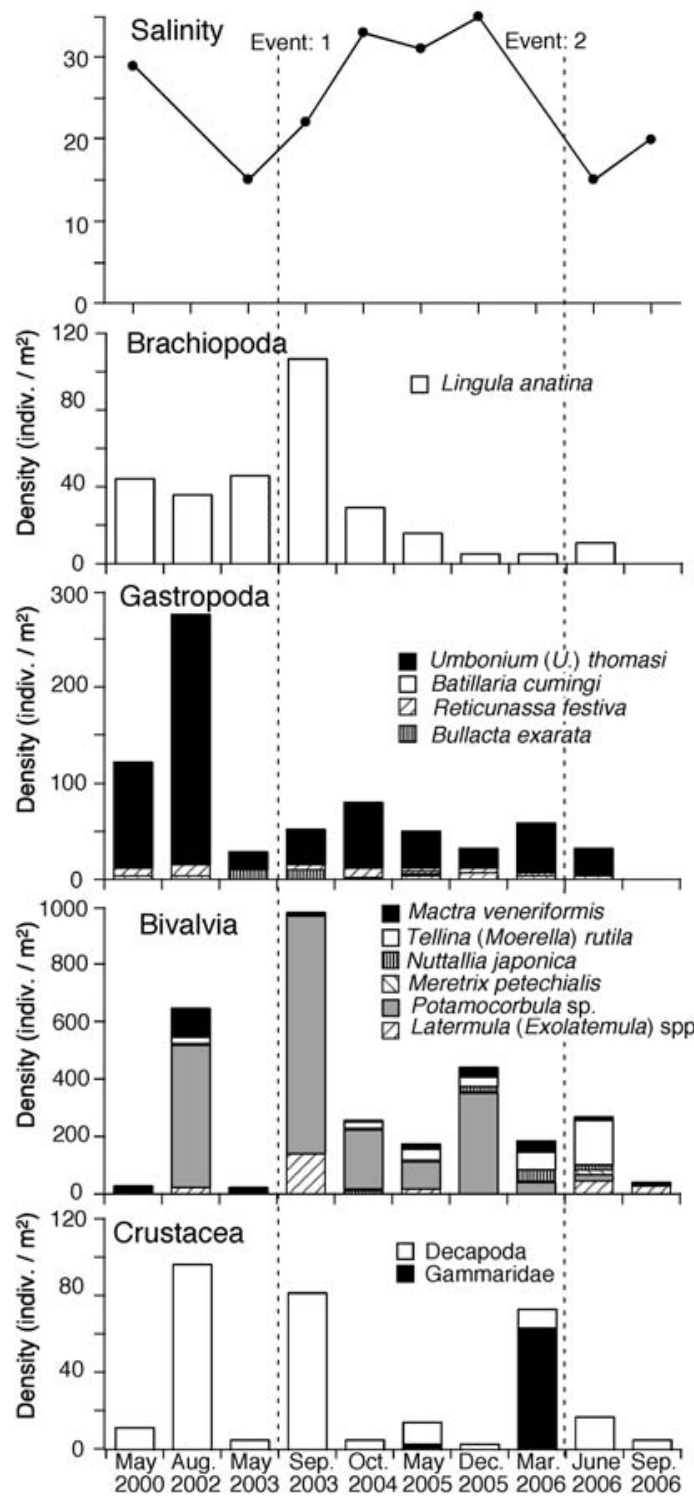

Fig. 2 Temporal changes in the salinity of surface water around sampling station 8 and mean individual densities of a brachiopod, gastropods, bivalves, and crustaceans collected from the tidal flats around

(A) Okbong-ri, Okseo-myeon, Gunsan City, and (B) Simpo-ri, Jinbong-myeon, Gimje City, between May 2000 and September 2006

Dotted lines show major events in the Saemangeum Reclamation Project: (1) the northern part of this dike was completed in June 2003, and (2) the southern part of the dike was completed in April 2006. 
(Philippi, 1849), and more than eight species of bivalves such as Mactra veneriformis Reeve (1854), Tellina (Moerella) rutila Dunker (1860), Meretrix petechialis (Lamarck, 1818) and Laternula (Exolaternula) spp. were collected from the tidal flats (Table 2). Populations of Umbonium (U.) thomasi and Tellina (Moerella) rutila were dense, at $>100$ individuals $/ \mathrm{m}^{2}$, in the stations between 1,000 and 2,000 $\mathrm{m}$ from land in the tidal flat around Okbong-ri (Table 2). Also, a unique ectosymbiotic bivalve species, Montacutidae gen. et sp. attached to the shell of Lingula anatina was found in quadrat samples in the tidal flat around Simpo-ri (see Table 2 ; Sato, 2006 ; Hong et al., 2007).

However, after the completion of the northern part of the reclamation dike in June 2003, rapid changes in bivalves and gastropods communities occurred in the tidal flats of the Saemangeum area. In the tidal flat around Okbongri, Umbonium (U.) thomasi and Tellina (Moerella) rutila decreased rapidly after May 2003, whereas the gastropod Batillaria cumingii (Crosse, 1862) and bivalves such as Felaniella sowerbyi Kuroda and Habe (1951) and Laternula (Exolaternula) spp. increased rapidly (Fig. 2-A). At the sampling stations where Umbonium (U.) thomasi was abundant in May 2000, the density of Batillaria cumingii increased since in May 2003 (Table 2). Felaniella sowerbyi and Laternula (Exolaternula) spp. were collected at stations between 1,000 and 1,200 $\mathrm{m}$ from land in May 2003 and October 2004, but they decreased rapidly after May 2005. Finally, after the completion of the southern part of the reclamation dike in April 2006, only three species of mollusks, Batillaria cumingii, Laternula (Exolaternula) spp. and Glauconome chinensis were alive on the dried tidal flat around Okbong-ri (Table 2).

In the tidal flat around Simpo-ri, many benthic animals such as mollusks, a brachiopod, and crustaceans also decreased rapidly after 2003 (Fig. 2-B). After this time, several species of bivalves, e.g., Potamocorbula sp. and Laternula (Exolaternula) spp. increased suddenly and decreased subsequently (Fig. 2-B). In particular, Potamocorbula sp. was not found in the tidal flat around Simpo-ri in May 2000, but it appeared suddenly at high densities starting in September 2001 (Sato, 2002, 2005). The maxi- mum density of this species was 7,936 individuals $/ \mathrm{m}^{2}$ at St. 2 , where was $200 \mathrm{~m}$ from land, in September 2003 (Table 2).

In addition to mollusks, the brachiopod Lingula anatina was collected abundantly from many stations on the tidal flat until September 2003 (Fig. 2). However, this species also decreased since October 2004 and finally disappeared in September 2006. Among the crustaceans, many crabs were found until September 2003, but they also decreased after October 2004 (Fig. 2). Instead of crabs, gammaridean amphipods, especially Corophium spp., increased suddenly from May 2005 to March 2006. However, they also decreased rapidly and disappeared in September 2006.

\section{Discussion}

An and Koh (1992) investigated benthos fauna in the Saemangeum area along eight transects on the tidal flats, including Okbong-ri and Simpori, in July 1988, before the construction of the dike. They found a total of 64 species of benthic animals, including mollusks, brachiopods, crustaceans, and polychaetous annelids, in 65 quadrat samples $(50 \times 100 \mathrm{~cm}$; two per station) by digging down to $30 \mathrm{~cm}$ in sediment depth. The mean density of these animals was 1,225 individuals $/ \mathrm{m}^{2}$. Je (2000) reported the benthic fauna from nine transects surveyed in July 1996, located in areas similar to those of An and Koh (1992). He listed 45 species of benthic animals from 45 quadrat samples $(50 \times 50 \mathrm{~cm}$; two per station), with a mean density of 214 individuals/ $\mathrm{m}^{2}$, and concluded that species numbers and densities had decreased during dike construction from 1988 to 1996.

Je (2000) also compiled data on the benthic animals collected from intertidal and subtidal zones around this area and reported 82 species of mollusks. In addition, Yamashita et al. (2006) recorded 121 species of mollusks from sea and brackish waters in the Saemangeum area. In particular, 50 species of mollusks were found between 2000 and 2003 in the tidal flat around Okbong-ri (Yamashita et al., 2006). However, most of these species rapidly decreased after 2003, and only a few species such as Batillaria cumingii and Glauconome chinensis remained alive on the dried tidal flat after the completion of all 
Table 2 Numbers of individual of benthic animals collected from quadrats at

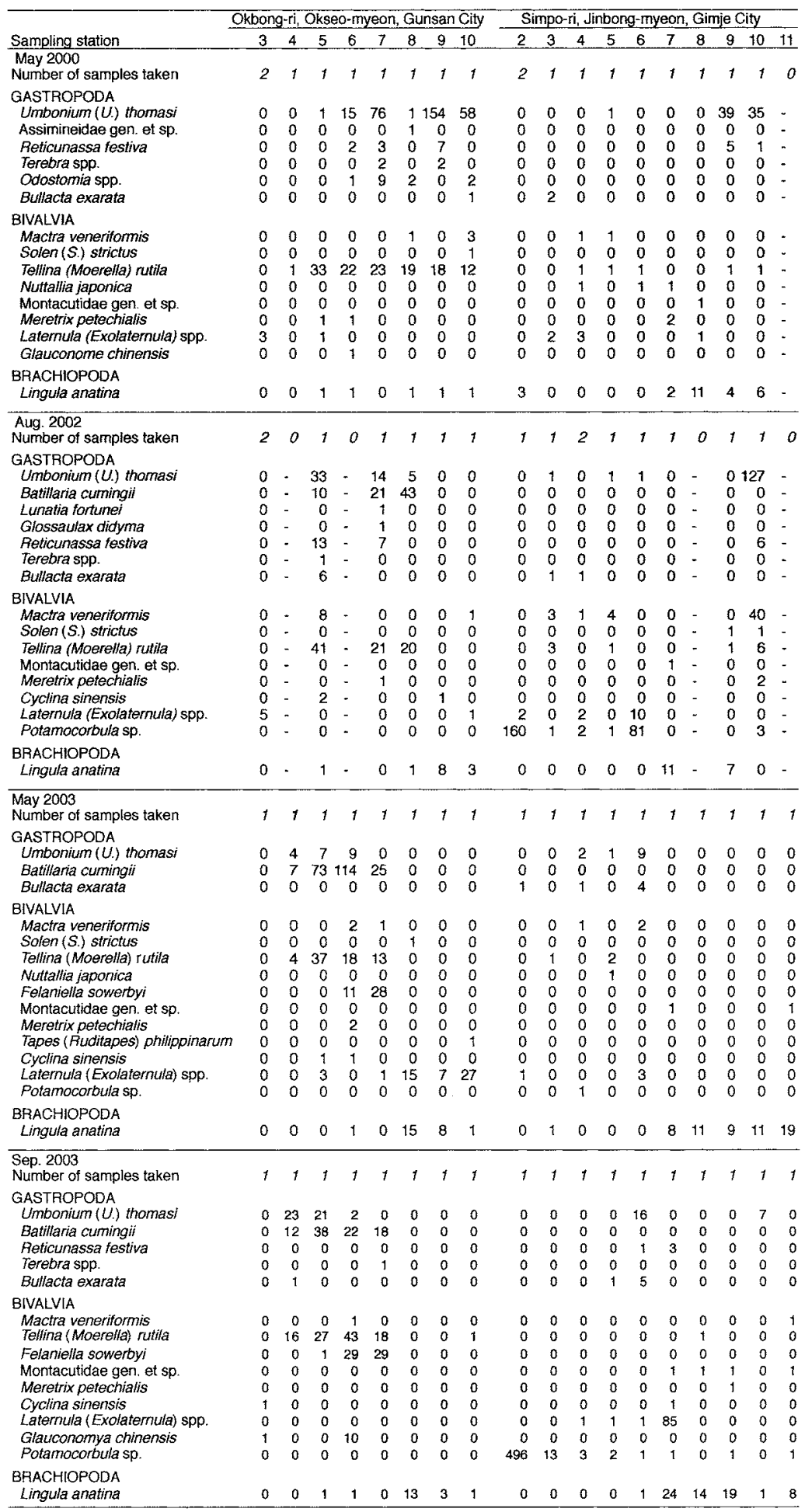


each sampling station in the tidal flat of the Saemangeum area, South Korea

\begin{tabular}{|c|c|c|c|c|c|c|c|c|c|c|c|c|c|c|c|c|c|c|}
\hline Sampling station & 3 & 4 & 5 & 6 & 7 & 8 & 9 & 10 & 2 & 3 & 4 & 5 & 6 & 7 & 8 & 9 & 10 & 11 \\
\hline $\begin{array}{l}\text { Oct. } 2004 \\
\text { Number of samples taken }\end{array}$ & 1 & 1 & 1 & 1 & 1 & 1 & 1 & 1 & 1 & 1 & 1 & 1 & 1 & 1 & 1 & 1 & 1 & 1 \\
\hline $\begin{array}{l}\text { GASTROPODA } \\
\text { Umbonium }(U) \text { thomasi } \\
\text { Batillaria cumingii } \\
\text { Reticunassa festiva } \\
\text { Bullacta exarata }\end{array}$ & $\begin{array}{l}0 \\
0 \\
0 \\
3\end{array}$ & $\begin{array}{l}0 \\
0 \\
0 \\
0\end{array}$ & $\begin{array}{r}1 \\
23 \\
0 \\
0\end{array}$ & $\begin{array}{l}0 \\
8 \\
0 \\
0\end{array}$ & $\begin{array}{r}0 \\
15 \\
0 \\
0\end{array}$ & $\begin{array}{l}0 \\
0 \\
0 \\
0\end{array}$ & $\begin{array}{l}0 \\
0 \\
0 \\
0\end{array}$ & $\begin{array}{l}0 \\
0 \\
0 \\
0\end{array}$ & $\begin{array}{l}0 \\
0 \\
0 \\
0\end{array}$ & $\begin{array}{l}0 \\
0 \\
0 \\
0\end{array}$ & $\begin{array}{l}0 \\
0 \\
0 \\
0\end{array}$ & $\begin{array}{l}8 \\
0 \\
0 \\
0\end{array}$ & $\begin{array}{l}2 \\
0 \\
0 \\
0\end{array}$ & $\begin{array}{l}5 \\
0 \\
1 \\
0\end{array}$ & $\begin{array}{l}7 \\
0 \\
0 \\
1\end{array}$ & $\begin{array}{l}4 \\
0 \\
0 \\
0\end{array}$ & $\begin{array}{r}12 \\
0 \\
5 \\
0\end{array}$ & $\begin{array}{l}5 \\
0 \\
0 \\
0\end{array}$ \\
\hline $\begin{array}{l}\text { BIVALLVIA } \\
\text { Mactra veneriformis } \\
\text { Tellina (Moerelia) rutila } \\
\text { Nuttallia japonica } \\
\text { Felaniella sowerbyi } \\
\text { Meretrix petechialis } \\
\text { Cyclina sinensis } \\
\text { Laternula (Exolaternula) spp. } \\
\text { Glauconomya chinensis } \\
\text { Potamocorbula sp. }\end{array}$ & $\begin{array}{l}0 \\
0 \\
0 \\
0 \\
0 \\
0 \\
0 \\
0 \\
0\end{array}$ & $\begin{array}{l}0 \\
1 \\
0 \\
0 \\
0 \\
0 \\
1 \\
0 \\
0\end{array}$ & $\begin{array}{l}0 \\
3 \\
0 \\
0 \\
0 \\
0 \\
0 \\
0 \\
0\end{array}$ & $\begin{array}{r}0 \\
0 \\
0 \\
2 \\
0 \\
0 \\
141 \\
1 \\
0\end{array}$ & $\begin{array}{r}0 \\
0 \\
0 \\
0 \\
0 \\
0 \\
15 \\
0 \\
0\end{array}$ & $\begin{array}{l}0 \\
0 \\
0 \\
0 \\
0 \\
1 \\
0 \\
0 \\
0\end{array}$ & $\begin{array}{l}0 \\
2 \\
0 \\
0 \\
0 \\
0 \\
0 \\
0 \\
0\end{array}$ & $\begin{array}{l}0 \\
0 \\
0 \\
0 \\
0 \\
1 \\
0 \\
0 \\
0\end{array}$ & $\begin{array}{r}0 \\
0 \\
0 \\
0 \\
0 \\
0 \\
0 \\
0 \\
74\end{array}$ & $\begin{array}{r}0 \\
0 \\
0 \\
0 \\
0 \\
0 \\
0 \\
0 \\
19\end{array}$ & $\begin{array}{r}1 \\
0 \\
1 \\
0 \\
0 \\
0 \\
0 \\
0 \\
34\end{array}$ & $\begin{array}{l}5 \\
2 \\
0 \\
0 \\
0 \\
0 \\
0 \\
0 \\
1\end{array}$ & $\begin{array}{l}0 \\
0 \\
0 \\
0 \\
0 \\
0 \\
1 \\
0 \\
0\end{array}$ & $\begin{array}{l}0 \\
3 \\
0 \\
0 \\
0 \\
0 \\
0 \\
0 \\
0\end{array}$ & $\begin{array}{l}0 \\
5 \\
0 \\
0 \\
1 \\
0 \\
1 \\
0 \\
0\end{array}$ & $\begin{array}{l}2 \\
4 \\
0 \\
0 \\
1 \\
0 \\
0 \\
0 \\
0\end{array}$ & $\begin{array}{l}4 \\
0 \\
0 \\
0 \\
0 \\
0 \\
0 \\
0 \\
0\end{array}$ & $\begin{array}{l}0 \\
0 \\
0 \\
0 \\
1 \\
0 \\
0 \\
0 \\
0\end{array}$ \\
\hline $\begin{array}{l}\text { BRACHIOPODA } \\
\text { Lingula anatina }\end{array}$ & 0 & 0 & 2 & 1 & 1 & 1 & 3 & 1 & 0 & 0 & 0 & 0 & 0 & 8 & 4 & 2 & 4 & 0 \\
\hline $\begin{array}{l}\text { May } 2005 \\
\text { Number of samples taken }\end{array}$ & 1 & 1 & 1 & 1 & 1 & 1 & 1 & 1 & 1 & 1 & 1 & 1 & 1 & 1 & 1 & 1 & 1 & 1 \\
\hline $\begin{array}{l}\text { GASTROPODA } \\
\text { Umbonium }(U) \text { thomasi } \\
\text { Batillaria cumingii } \\
\text { Lunatia fortumei } \\
\text { Reticunassa festiva } \\
\text { Terebra spp. } \\
\text { Bullacta exarata }\end{array}$ & $\begin{array}{l}0 \\
0 \\
0 \\
0 \\
0 \\
0\end{array}$ & $\begin{array}{r}0 \\
16 \\
0 \\
0 \\
0 \\
0\end{array}$ & $\begin{array}{r}0 \\
23 \\
0 \\
0 \\
0 \\
0\end{array}$ & $\begin{array}{r}0 \\
25 \\
0 \\
0 \\
1 \\
0\end{array}$ & $\begin{array}{r}0 \\
15 \\
0 \\
0 \\
0 \\
0\end{array}$ & $\begin{array}{l}0 \\
0 \\
0 \\
1 \\
0 \\
0\end{array}$ & $\begin{array}{l}0 \\
0 \\
0 \\
1 \\
0 \\
0\end{array}$ & $\begin{array}{l}0 \\
0 \\
0 \\
1 \\
0 \\
0\end{array}$ & $\begin{array}{l}0 \\
0 \\
0 \\
0 \\
0 \\
1\end{array}$ & $\begin{array}{l}0 \\
0 \\
0 \\
0 \\
0 \\
0\end{array}$ & $\begin{array}{l}0 \\
0 \\
0 \\
0 \\
0 \\
0\end{array}$ & $\begin{array}{l}0 \\
0 \\
0 \\
0 \\
0 \\
0\end{array}$ & $\begin{array}{l}0 \\
0 \\
0 \\
0 \\
0 \\
0\end{array}$ & $\begin{array}{l}0 \\
0 \\
2 \\
0 \\
0 \\
1\end{array}$ & $\begin{array}{r}16 \\
0 \\
0 \\
1 \\
0 \\
0\end{array}$ & $\begin{array}{l}2 \\
0 \\
0 \\
0 \\
0 \\
0\end{array}$ & $\begin{array}{l}6 \\
0 \\
0 \\
1 \\
1 \\
0\end{array}$ & $\begin{array}{l}1 \\
0 \\
0 \\
0 \\
0 \\
0\end{array}$ \\
\hline $\begin{array}{l}\text { BIVALVIA } \\
\text { Mactra veneriformis } \\
\text { Solen (S.) strictus } \\
\text { Tellina (Moerella) rutila } \\
\text { Felaniella sowerbyi } \\
\text { Meretrix petechialis } \\
\text { Laternula (Exolaternula) spp. } \\
\text { Potamocorbula sp. }\end{array}$ & $\begin{array}{l}0 \\
0 \\
0 \\
0 \\
0 \\
0 \\
0\end{array}$ & $\begin{array}{l}0 \\
0 \\
0 \\
0 \\
0 \\
2 \\
0\end{array}$ & $\begin{array}{l}0 \\
0 \\
0 \\
0 \\
0 \\
0 \\
0\end{array}$ & $\begin{array}{l}0 \\
0 \\
8 \\
2 \\
0 \\
0 \\
0\end{array}$ & $\begin{array}{l}0 \\
0 \\
0 \\
0 \\
0 \\
1 \\
0\end{array}$ & $\begin{array}{l}0 \\
0 \\
1 \\
1 \\
0 \\
0 \\
0\end{array}$ & $\begin{array}{l}0 \\
0 \\
0 \\
0 \\
0 \\
0 \\
0\end{array}$ & $\begin{array}{l}0 \\
0 \\
0 \\
0 \\
0 \\
0 \\
0\end{array}$ & $\begin{array}{l}0 \\
0 \\
0 \\
0 \\
0 \\
1 \\
1\end{array}$ & $\begin{array}{l}0 \\
0 \\
1 \\
0 \\
0 \\
1 \\
1\end{array}$ & $\begin{array}{l}0 \\
0 \\
0 \\
0 \\
0 \\
5 \\
2\end{array}$ & $\begin{array}{r}0 \\
0 \\
0 \\
0 \\
2 \\
1 \\
55\end{array}$ & $\begin{array}{l}0 \\
0 \\
2 \\
0 \\
0 \\
0 \\
0\end{array}$ & $\begin{array}{l}0 \\
1 \\
5 \\
0 \\
1 \\
1 \\
0\end{array}$ & $\begin{array}{l}1 \\
0 \\
5 \\
0 \\
0 \\
0 \\
0\end{array}$ & $\begin{array}{r}4 \\
0 \\
10 \\
0 \\
0 \\
0 \\
0\end{array}$ & $\begin{array}{l}4 \\
0 \\
3 \\
0 \\
1 \\
0 \\
0\end{array}$ & $\begin{array}{l}0 \\
0 \\
0 \\
0 \\
0 \\
0 \\
0\end{array}$ \\
\hline $\begin{array}{l}\text { BRACHIOPODA } \\
\text { Lingula anatina }\end{array}$ & 0 & 0 & 0 & 0 & 1 & 5 & 0 & 1 & 0 & 0 & 0 & 1 & 0 & 2 & 1 & 5 & 1 & 0 \\
\hline $\begin{array}{l}\text { Mar. } 2006 \\
\text { Number of samples taken }\end{array}$ & 1 & 1 & 1 & 1 & 1 & 1 & 1 & 1 & 1 & 1 & 7 & 1 & 1 & 1 & 1 & 1 & 1 & 1 \\
\hline $\begin{array}{l}\text { GASTROPODA } \\
\text { Umbonium }(U \text { ) thomasi } \\
\text { Batillaria cumingii } \\
\text { Reficunassa festiva } \\
\text { Assimineidae gen. et sp. } \\
\text { Bullacta exarata }\end{array}$ & $\begin{array}{l}0 \\
0 \\
0 \\
0 \\
0\end{array}$ & $\begin{array}{l}0 \\
0 \\
0 \\
0 \\
0\end{array}$ & $\begin{array}{r}0 \\
45 \\
0 \\
0 \\
0\end{array}$ & $\begin{array}{r}0 \\
38 \\
0 \\
0 \\
0\end{array}$ & $\begin{array}{l}0 \\
6 \\
0 \\
0 \\
0\end{array}$ & $\begin{array}{r}0 \\
20 \\
0 \\
0 \\
0\end{array}$ & $\begin{array}{l}0 \\
0 \\
0 \\
0 \\
0\end{array}$ & $\begin{array}{l}0 \\
0 \\
0 \\
0 \\
0\end{array}$ & $\begin{array}{l}0 \\
0 \\
0 \\
0 \\
0\end{array}$ & $\begin{array}{l}0 \\
0 \\
0 \\
0 \\
0\end{array}$ & $\begin{array}{l}0 \\
0 \\
0 \\
0 \\
0\end{array}$ & $\begin{array}{l}0 \\
0 \\
0 \\
0 \\
0\end{array}$ & $\begin{array}{l}0 \\
0 \\
0 \\
0 \\
0\end{array}$ & $\begin{array}{l}0 \\
0 \\
0 \\
0 \\
1\end{array}$ & $\begin{array}{l}4 \\
0 \\
0 \\
0 \\
0\end{array}$ & $\begin{array}{l}0 \\
0 \\
0 \\
0 \\
0\end{array}$ & $\begin{array}{l}9 \\
0 \\
1 \\
0 \\
0\end{array}$ & $\begin{array}{r}19 \\
0 \\
1 \\
1 \\
1\end{array}$ \\
\hline $\begin{array}{l}\text { BIVALVIA } \\
\text { Mactra veneriformis } \\
\text { Solen (S.) strictus } \\
\text { Tellina (Moerella) rutila } \\
\text { Nuttallia japonica } \\
\text { Meretrix petechialis } \\
\text { Cyclina sinensis } \\
\text { Glauconomya chinensis } \\
\text { Potamocorbula sp. }\end{array}$ & $\begin{array}{l}0 \\
0 \\
0 \\
0 \\
0 \\
0 \\
0 \\
0\end{array}$ & $\begin{array}{l}0 \\
0 \\
0 \\
0 \\
0 \\
0 \\
0 \\
0\end{array}$ & $\begin{array}{l}0 \\
0 \\
1 \\
0 \\
0 \\
1 \\
2 \\
0\end{array}$ & $\begin{array}{l}0 \\
0 \\
0 \\
0 \\
0 \\
0 \\
4 \\
0\end{array}$ & $\begin{array}{l}0 \\
2 \\
0 \\
0 \\
0 \\
0 \\
2 \\
0\end{array}$ & $\begin{array}{l}0 \\
0 \\
0 \\
0 \\
0 \\
0 \\
0 \\
0\end{array}$ & $\begin{array}{l}0 \\
0 \\
0 \\
0 \\
0 \\
0 \\
0 \\
0\end{array}$ & $\begin{array}{l}0 \\
0 \\
0 \\
0 \\
0 \\
0 \\
0 \\
0\end{array}$ & $\begin{array}{l}0 \\
0 \\
0 \\
0 \\
0 \\
0 \\
0 \\
3\end{array}$ & $\begin{array}{l}2 \\
1 \\
3 \\
0 \\
0 \\
0 \\
0 \\
6\end{array}$ & $\begin{array}{l}0 \\
0 \\
1 \\
0 \\
0 \\
0 \\
0 \\
0\end{array}$ & $\begin{array}{l}7 \\
0 \\
4 \\
0 \\
0 \\
0 \\
0 \\
2\end{array}$ & $\begin{array}{l}1 \\
0 \\
1 \\
4 \\
0 \\
0 \\
0 \\
1\end{array}$ & $\begin{array}{l}2 \\
0 \\
0 \\
4 \\
1 \\
0 \\
0 \\
\mathbf{3}\end{array}$ & $\begin{array}{r}2 \\
0 \\
13 \\
1 \\
1 \\
0 \\
0 \\
3\end{array}$ & $\begin{array}{r}5 \\
0 \\
1 \\
13 \\
2 \\
0 \\
0 \\
2\end{array}$ & $\begin{array}{l}1 \\
0 \\
2 \\
0 \\
1 \\
0 \\
0 \\
2\end{array}$ & $\begin{array}{r}2 \\
1 \\
14 \\
1 \\
1 \\
0 \\
0 \\
1\end{array}$ \\
\hline $\begin{array}{l}\text { BRACHIOPODA } \\
\text { lingula anatina }\end{array}$ & 0 & 0 & 0 & 0 & 2 & 2 & 5 & 2 & 0 & 0 & 0 & 0 & 1 & 0 & 0 & 0 & 0 & 2 \\
\hline $\begin{array}{l}\text { June } 2006 \\
\text { Number of samples taken }\end{array}$ & $1^{*}$ & $1^{*}$ & 1 & 1 & 1 & 1 & 0 & 0 & 1 & 1 & 1 & 1 & 1 & 1 & 1 & 1 & 1 & 1 \\
\hline $\begin{array}{l}\text { GASTROPODA } \\
\text { Umbonium }(\text { U.) thomasi } \\
\text { Batillaria cumingii } \\
\text { Hima festiva } \\
\text { Bullacta exarata }\end{array}$ & $\begin{array}{l}0 \\
0 \\
0 \\
0\end{array}$ & $\begin{array}{l}0 \\
0 \\
0 \\
0\end{array}$ & $\begin{array}{r}0 \\
45 \\
0 \\
0\end{array}$ & $\begin{array}{l}0 \\
7 \\
0 \\
0\end{array}$ & $\begin{array}{l}0 \\
0 \\
0 \\
0\end{array}$ & $\begin{array}{l}0 \\
0 \\
0 \\
0\end{array}$ & $\begin{array}{l}- \\
-\end{array}$ & $\begin{array}{l}- \\
- \\
-\end{array}$ & $\begin{array}{l}0 \\
0 \\
0 \\
1\end{array}$ & $\begin{array}{l}0 \\
0 \\
0 \\
1\end{array}$ & $\begin{array}{l}0 \\
0 \\
0 \\
0\end{array}$ & $\begin{array}{l}0 \\
0 \\
0 \\
0\end{array}$ & $\begin{array}{l}0 \\
0 \\
0 \\
0\end{array}$ & $\begin{array}{l}1 \\
0 \\
1 \\
1\end{array}$ & $\begin{array}{r}14 \\
0 \\
0 \\
0\end{array}$ & $\begin{array}{l}2 \\
0 \\
0 \\
0\end{array}$ & $\begin{array}{l}0 \\
0 \\
0 \\
0\end{array}$ & $\begin{array}{l}0 \\
0 \\
0 \\
0\end{array}$ \\
\hline $\begin{array}{l}\text { BIVALVIA } \\
\text { Mactra veneriformis } \\
\text { Tellina (Moerella) rutila } \\
\text { Nuttallia japonica } \\
\text { Corbicula (C) japonica } \\
\text { Meretrix petechialis } \\
\text { Laternula (Exolatemula) spp. } \\
\text { Glauconomya chinensis } \\
\text { Potamocorbula sp. }\end{array}$ & $\begin{array}{l}0 \\
0 \\
0 \\
0 \\
0 \\
0 \\
1 \\
0\end{array}$ & $\begin{array}{l}0 \\
0 \\
0 \\
0 \\
0 \\
0 \\
0 \\
0\end{array}$ & $\begin{array}{l}0 \\
0 \\
0 \\
0 \\
0 \\
0 \\
3 \\
0\end{array}$ & $\begin{array}{l}0 \\
0 \\
0 \\
0 \\
0 \\
0 \\
4 \\
0\end{array}$ & $\begin{array}{l}0 \\
0 \\
0 \\
0 \\
0 \\
0 \\
0 \\
0\end{array}$ & $\begin{array}{l}0 \\
0 \\
0 \\
0 \\
0 \\
0 \\
0 \\
0\end{array}$ & $\begin{array}{l}- \\
- \\
- \\
- \\
- \\
-\end{array}$ & $\begin{array}{l}- \\
- \\
- \\
- \\
- \\
- \\
-\end{array}$ & $\begin{array}{l}0 \\
1 \\
0 \\
0 \\
0 \\
2 \\
0 \\
0\end{array}$ & $\begin{array}{r}1 \\
0 \\
0 \\
0 \\
0 \\
16 \\
0 \\
0\end{array}$ & $\begin{array}{l}0 \\
3 \\
1 \\
0 \\
0 \\
0 \\
0 \\
1\end{array}$ & $\begin{array}{l}3 \\
1 \\
0 \\
0 \\
0 \\
3 \\
0 \\
0\end{array}$ & $\begin{array}{r}0 \\
34 \\
3 \\
0 \\
0 \\
4 \\
0 \\
2\end{array}$ & $\begin{array}{l}0 \\
7 \\
1 \\
0 \\
0 \\
0 \\
0 \\
2\end{array}$ & $\begin{array}{r}1 \\
11 \\
0 \\
0 \\
10 \\
0 \\
0 \\
0\end{array}$ & $\begin{array}{r}1 \\
11 \\
4 \\
1 \\
0 \\
1 \\
0 \\
5\end{array}$ & $\begin{array}{r}1 \\
22 \\
0 \\
0 \\
0 \\
0 \\
0 \\
2\end{array}$ & $\begin{array}{l}0 \\
7 \\
1 \\
0 \\
0 \\
3 \\
0 \\
3\end{array}$ \\
\hline $\begin{array}{l}\text { BRACHIOPODA } \\
\text { Lingula anatina }\end{array}$ & 0 & 0 & 0 & 0 & 0 & 0 & - & - & 0 & 0 & 0 & 0 & 0 & 0 & 0 & 0 & 7 & 0 \\
\hline $\begin{array}{l}\text { September } 2006 \\
\text { Number of samples taken }\end{array}$ & $7^{*}$ & $1^{*}$ & 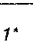 & 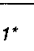 & $1^{*}$ & 1 & 0 & 0 & 1 & 1 & 1 & 1 & 1 & 1 & 1 & 1 & 1 & 0 \\
\hline $\begin{array}{l}\text { GASTROPODA } \\
\text { Batillaria cumingii }\end{array}$ & 0 & 0 & 0 & 3 & 7 & 0 & - & - & 0 & 0 & 0 & 0 & 0 & 0 & 0 & 0 & 0 & - \\
\hline $\begin{array}{l}\text { BIVALVIA } \\
\text { Mactra veneriformis } \\
\text { Tellina (Moerella) rutila } \\
\text { Laternula (Exolatemula) spp. } \\
\text { Glauconomya chinensis }\end{array}$ & $\begin{array}{l}0 \\
0 \\
0 \\
0\end{array}$ & $\begin{array}{l}0 \\
0 \\
0 \\
0\end{array}$ & $\begin{array}{l}0 \\
0 \\
0 \\
0\end{array}$ & $\begin{array}{l}0 \\
0 \\
0 \\
0\end{array}$ & $\begin{array}{l}0 \\
0 \\
0 \\
0\end{array}$ & $\begin{array}{l}0 \\
0 \\
1 \\
0\end{array}$ & $\begin{array}{l}- \\
-\end{array}$ & $\begin{array}{l}- \\
- \\
-\end{array}$ & $\begin{array}{l}0 \\
0 \\
4 \\
0\end{array}$ & $\begin{array}{r}0 \\
0 \\
14 \\
0\end{array}$ & $\begin{array}{l}0 \\
0 \\
5 \\
0\end{array}$ & $\begin{array}{l}0 \\
0 \\
2 \\
1\end{array}$ & $\begin{array}{l}0 \\
0 \\
2 \\
0\end{array}$ & $\begin{array}{l}0 \\
0 \\
1 \\
0\end{array}$ & $\begin{array}{l}0 \\
0 \\
0 \\
0\end{array}$ & $\begin{array}{l}0 \\
1 \\
0 \\
0\end{array}$ & $\begin{array}{l}1 \\
2 \\
0 \\
0\end{array}$ & $\begin{array}{l}- \\
-\end{array}$ \\
\hline
\end{tabular}

* Living animals were collected from the dried tidal flat without sieving. 
dikes (Table 2).

In Isahaya Bay, the gastropod Cerithideopsilla djadjariensis (K. Martin, 1899) inhabited the middle part of the dried mud flat until 1 year after isolation, and gastropods distributed in reedy marshes, e.g., Cerithidea rhizophorarum A. Adams (1855) and Pseudomphala miyazakii (Habe, 1943), persisted until 2.5 years after isolation (Sato, 2001). Several bivalves such as Glauconome chinensis and Estellacar olivacea (Reeve, 1844) were also found living on the upper part of the dried mud flat in July 1997 (Sato, 1997, 2001). Thus, it appears that species of both gastropods and bivalves distributed in the upper and middle parts of tidal flat are more tolerant to desiccation and starvation than those inhabiting the lower part.

In the subtidal zone (adjustment pond) of Isahaya Bay, most species of marine mollusks died off simultaneously when the salinity of the bottom water decreased $<5$ psu (Sato et al., 2001 ; Sato and Azuma, 2002). In the Saemangeum area, the salinity did not decrease below 10 psu until September 2006 (Fig. 2) because the water gates were still opened. Therefore, small numbers of benthic animals could remain alive on the tidal flat around Simpo-ri. However, if the water gates remain closed, the salinity will rapidly decrease to $<5 \mathrm{psu}$, and most marine animals will be lost completely from the Saemangeum area.

Some brackish species such as Potamocorbula sp. and Corophium spp. temporarily increased in the tidal flat around Saemangeum after the isolation of the northern dike (Fig. 2). In July 1988, only a few individuals of Potamocorbula sp. and gammaridean amphipods were collected from the intertidal zone around the Saemangeum area (An and Koh, 1992). Potamocorbula sp. was collected from the tidal flat around Simpo-ri in August 1992 with a mean density of 17 individuals $/ \mathrm{m}^{2}$, but it was not a dominant species in July 1996 (Je, 2000). This species was also not found in May 2000, but it appeared suddenly at high densities beginning September 2001 (Sato, 2002, 2005). In addition, Corophium spp. was not a dominant species until July 1996 in tidal flat around the Saemangeum area (Je, 2000), but temporarily increased in March 2006 (Table 2).
In Isahaya Bay, Potamocorbula sp. and Corophium sinense Zhang (1974) also increased rapidly after the completion of the dike (Azuma, 2000 ; Sato et al., 2006). These species increased rapidly when the salinity of the bottom water became $<10$ psu (Sato et al., 2006) ; in particular, Potamocorbula sp. was distributed at stations where the salinity of the bottom water was $<5$ psu (Sato et al., 2001). Moreover, Corophium sinense also increased after completion of the dike in the Sihwa Reclamation Project along the northern coast on South Korea when the salinity of the bottom water decreased bellow 10 psu (Hong et al., 1997). Therefore, these species are able to survive and become very abundant following environmental disturbance such as isolation (Sato, 2002). These results suggest that the responses of benthic animals after isolation in the Yellow Sea and Ariake Bay were similar, and that the situations observed in Isahaya Bay and Sihwa Lake are now likely to be mirrored in the Saemangeum area.

Changes in benthic fauna were confirmed not only inside the dike, but also in the outer parts of Isahaya Bay and Sihwa Lake after the completion of the dikes. In Isahaya Bay, red tides and hypoxic water have been observed frequently, and many bivalve species have decreased rapidly since 1997 (Tsustumi, 2006). Kanazawa et al. (2005) pointed out that temporal changes in bivalve fauna were strongly affected by the environmental changes such as the occurrence of hypoxic water and changes in the grain size of bottom sediments, and that those were caused by the isolation of Isahaya Bay. Moreover, the Sihwa Reclamation Project was halted in 1996 because of its negative effects on the environment; the water gates are now open to introduce seawater inside the dike. Changes in benthic fauna have been also confirmed in the outer parts of the Saemangeum dike (Sato, personal observation). These facts suggest that the Saemangeum dike will negatively affect the Yellow Sea in the near future.

\section{Acknowledgments}

We are grateful to Mr. Dong-Pil Oh, Mr. KilWook Yoe, Mr. Yung-Ki Ju, Mr. Kuijae Bae and members of the Japan/Korea Tidal-flats Joint Survey Group for their help in collecting sam- 
ples. This work was supported by the Toyota Foundation, Takagi Fund for Citizen Science, Kurita Water and Environment Foundation, and Grant-in-Aid for Scientific Research from the Japan Society for the Promotion of Science (No. 15740308 for S. Sato).

\section{References}

An, S.M. and Koh, C.H. (1992) Environments and distribution of benthic animals on the MangyungDongjin tidal flat, west coast of Korea. The Journal of the Oceanological Society of Korea, 27, 78-90. (K $+\mathrm{E})$

Azuma, M. (2000) Chapter 13 Effect of the Isahaya Bay Reclamation Project. Sato, M. (ed.) Life in Ariake Sea : Biodiversity in tidal flats and estuaries : $320-$ 337, Kaiyu-sha Press. (J)

Hong, J.S. (2000) Chapter 11 Benthos of the tidal flats in Korea. Sato, M. (ed.) Life in Ariake Sea : Biodiversity in tidal flats and estuaries : 285-301, Kaiyusha Press. (J)

Hong, J.S., Jung, R.H., Seo, I.S., Yoon, K.T., Choi, B.M. and Yoo, J.W. (1997) How are the spatio-temporal distribution patterns of benthic macrofaunal communitites affected by the construction of Shihwa dike in the west coast of Korea? Journal of the Korean Fisheries Society, 30, 882-895. (K+E)

Hong, J.S., Yamashita, H. and Sato, S. (2007) The Saemangeum Reclamation Project in South Korea threatens to collapse a unique mollusk, ectosymbiotic bivalve species attached to the shell of Lingula anatina. Plankton \& Benthos Research, 2, 70-75.

Je, J.G. (2000) Marine benthic animals. Joint Survey Group for the Environment Changes caused by the Saemangeum Reclamation Project (ed.) Reports of the Joint Survey Group for the Environment Changes caused by the Saemangeum Reclamation Project : $92 \mathrm{p}$, Joint Survey Group for the Environment Changes caused by the Saemangeum Reclamation Project. (K)

Kanazawa, T., Sato, S., Azuma, M., Kondo, H., Nishinokubi, H. and Matsuo, M. (2005) Faunal change of bivalves in Ariake Bay after the construction of a dike for the reclamation of Isahaya Bay, western Kyushu, Japan. Japanese Journal of Benthology, 60, 30-42. $(\mathrm{J}+\mathrm{E})$

Sato, M. and Koh, C.H. (2004) Chapter 9 Biological richness of the Asian tidal flats and its crisis by human impact. Hong, S.K., Lee, J.A., Ihm, B.S., Farina, A., Son, Y., Kim, E.S. and Choe, J.C. (eds.) Ecological issues in a changing world-status, response and strategy : 135-155, Kluwer Academic Publishers.

Sato, S. (1997) The Isahaya Bay Reclamation Project: Ecology of mollusks. SCIaS (Asahi Kagaku), 24, 7475. $(\mathrm{J})$

Sato, S. (2000a) Actual paleoecology in the tidal flat of Isahaya Bay after reclamation (Pictorial). The Jour- nal of the Geological Society of Japan, 106, V-VI. (J) Sato, S. (2000b) Chapter 7 Bivalves. Sato, M. (ed.) Life in Ariake Sea : Biodiversity in tidal flats and estuaries : 150-183, Kaiyu-sha Press. (J)

Sato, S. (2001) Spatial distributions of bivalves and gastropods on the tidal mud flat created by the construction of a dike for reclamation of Isahaya Bay, western Kyushu, Japan. The Quaternary Research (Daiyonki-Kenkyu), 40, 43-51. (J+E)

Sato, S. (2002) Faunal response of bivalves and gastropods to large environmental disturbances caused by the construction of dyke for reclamation. Japanese Journal of Benthology, 57, 106-118. (J+E)

Sato, S. (2005) Faunal response of bivalves to large environmental disturbances caused by huge reclamation projects in Japan and Korea-a comparison with the fossil bivalve assemblages. Aquabiology, 27, 359-361. (J+E)

Sato, S. (2006) Drastic change of bivalves and gastropods caused by the huge reclamation projects in Japan and Korea. Plankton \& Benthos Research, 1, $123-137$.

Sato, S. and Azuma, M. (2002) Ecological and paleoecological implications of the rapid increase and decrease of an introduced bivalve Potamocorbula sp. after the construction of a dike for reclamation in the Isahaya Bay, Western Kyushu, Japan. Palaeogeography, Palaeoclimatology, Palaeoecology, 185, 369-378.

Sato, S., Azuma, M., Kondo, H. and Nishinokubi, H. (2001) Temporal change of bivalves and gastropods in Isahaya Bay shut off from Ariake Sea, western Kyushu, Japan. The Quaternary Research (DaiyonkiKenkyu), 40, 85-95. (J+E)

Sato, S. and Kanazawa, T. (2004) Faunal change of bivalves in Ariake Sea after the construction of the dike for reclamation in Isahaya Bay, western Kyushu, Japan. Fossils (Palaeontological Society of Japan), 76, 90-99. (J+E)

Sato, S., Sato, M., Matsuo, M. and Ichikawa, T. (2006) Conservation ecological research on "Disaster of the Ariake Sea" caused by the Isahaya Reclamation Project. Granted project report of the Takagi Fund for Citizen Science, 3, 43-49. (J)

Tsutsumi, H. (2006) Critical events in the Ariake Bay ecosystem : clam population collapse, red tides, and hypoxic bottom water. Plankton \& Benthos Research, 1, 3-25.

Wada, K., Nishihira, M., Furota, T., Nojima, S., Yamanishi, R., Nishikawa, T., Goshima, S., Suzuki, T., Kato, M., Shimamura, K. and Fukuda, H. (1996) Present status of estuarine locales and benthic invertebrates occurring in estuarine environment in Japan. WWF Japan Science Report, 3, 1-182. (J)

Yamashita, H., Sato, S., Mizuma, Y. and Nawa, J. (2006) A catalogue of marine mollusks in Saemangeum area. Japan/Korea Tidal-flats Joint Survey Group (ed.) Reports of Japan/Korea Tidal-flats Joint Survey Group in 2006 : 97-119, Japan/Korea Tidal-flats 
Joint Survey Group. (J)

(J) in Japanese, $(\mathrm{J}+\mathrm{E})$ in Japanese with English ab- stract, $(\mathrm{K})$ in Korean, $(\mathrm{K}+\mathrm{E})$ in Korean with English abstract.

\title{
海域開発に伴う沿岸域生物群集の変化 : 韓国セマングム 海域における大規模干拓堤防建設に伴う底生 生物群集の変化に関する事例研究
}

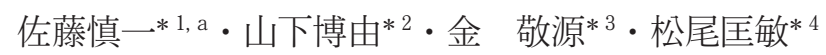

\begin{abstract}
〔要旨〕
大規模干拓事業は，世界中の沿岸域の自然環境に大き な影響をもたらしている。本研究では, 韓国西海岸のセ マングム海域と日本の諫早湾に扔いて, 干拓堤防建設に 伴う底生生物相の変化を調查・比較した. セマングム海 域では, 2006 年 4 月に世界最大の干拓堤防が完成した後 は, 干潟が徐々に乾燥を始好り，2006 年 6 月には多 くの貝類や他の底生生物の遺骸が出現した。諫早湾で は, 1997 年 4 月に干拓堤防が完成し, 有明湾からの海水

流入が遮断された。ここであ, 潮止め後数力月で潮間帯 が完全に乾燥し, 無数の貝類遺骸が乾燥した泥干潟に出 現した。底生生物相の变化は, 諫早湾の堤防外側でも確 認された。赤潮や貧酸素水塊が頻発し, 多くの二枚貝類 が 1997 年以降に急激に減少した. 同様の変化は, セマン グム干拓堤防外側であすでに確認されている。これらの 結果は, セマングム干拓事業が近い将来, 黄海の環境に 悪影響を及ぼすことを示唆している。
\end{abstract}

キーワード : 底生生物, 大規模干拓, 諫早湾, セマングム, 韓国

*1 東北大学総合学術博物館 =980-8578 仙台市青葉区荒巻字青葉 6-3.

*2 具類多様性研究所 $\overline{\mathbf{T}} 215-0038$ 藤沢市鵠沼松が岡 3-1-26-103.

*3 韓国環境運動連合 251 Nuha-dong, Jongno-gu, Seoul 110-806, South Korea.

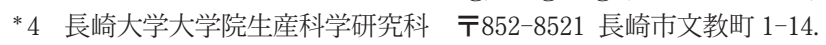

*a Corresponding author : kurosato@mail.tains.tohoku. ac.jp 